\title{
Placebo Effect in Clinical Trial Design for Irritable Bowel Syndrome
}

\author{
Eric Shah and Mark Pimentel* \\ GI Motility Program, Cedars-Sinai Medical Center, Los Angeles, CA, USA
}

Ongoing efforts to improve clinical trial design in irritable bowel syndrome have been hindered by high placebo response rates and ineffective outcome measures. We assessed established strategies to minimize placebo effect as well as the various approaches to placebo effect which can affect trial design. These include genetic markers such as catechol-0-methyltransferase, opioidergic and dopaminergic neurobiologic theory, pre-cebo effect centered on expectancy theory, and side effect unblinding grounded on conditioning theory. We reviewed endpoints used in the study of IBS over the past decade including adequate relief and subjective global relief, emphasizing their weaknesses in fully evaluating the IBS condition, specifically their motility effects based on functional net value and relative benefit-harm based on dropouts due to adverse events. The focus of this review is to highlight ongoing efforts to improve clinical trial design which can lead to better outcomes in a real-world setting. (J Neurogastroenterol Motil 2014;20:163-170)

\section{Key Words}

Clinical trial; Irritable bowel syndrome; Placebos

\section{Introduction}

Irritable bowel syndrome (IBS) is a non-lethal condition which affects a substantial proportion of the population. Based on the poor understanding of the pathophysiology of IBS, there is a significant unmet need for treatments. However, treatment in IBS is complicated by a number of factors, most importantly the poor and subjective outcome measures which are associated with a high placebo response rate in clinical trials. This culminates in the need for a large number of subjects in IBS trials to achieve significance. While subjective outcome measures are one aspect of the placebo effect, there are many factors that lead placebo ef- fects in IBS. In this review, we discuss pertinent issues to the placebo effect and trial design in IBS.

\section{Placebo Response and the Placebo Effect}

The role of placebo therapy in a randomized clinical trial is to delineate the therapeutic benefit attributable to a physiologic property of a drug which the placebo does not share. Thus, placebo therapy generally carries a negative implication in clinical trials against which drug response must be corrected. ${ }^{1,2}$ The placebo response rate in clinical trials against endpoints designed to evaluate investigational drugs in IBS is remarkable at $37.5 \%^{3}$ yet is

Received: September 29, 2013 Revised: October 26, 2013 Accepted: November 3, 2013

(c) This is an Open Access article distributed under the terms of the Creative Commons Attribution Non-Commercial License (http://creativecommons. org/licenses/by-nc/3.0) which permits unrestricted non-commercial use, distribution, and reproduction in any medium, provided the original work is properly cited.

*Correspondence: Mark Pimentel, MD, FRCP (C)

Director, GI Motility Program, Cedars-Sinai Medical Center, 8730 Alden Drive, Suite 201E, Los Angeles, California 90048, USA

Tel: +1-310-423-6143, Fax: +1-310-423-8356, E-mail: pimentelm@cshs.org

Financial support: None.

Conflicts of interest: Eric Shah has no competing interests. Mark Pimentel consults for Salix Pharmaceuticals and is on Dyax advisory board. 
not appreciably different than that found in influential studies of post-operative placebo analgesia conducted over 60 years ago. ${ }^{4}$ While direct clinical application of inert placebo remains in question, ${ }^{5}$ this high raw result warrants further study due to its implications on an effective evaluation of drug efficacy in IBS. ${ }^{6}$

At a basic level, placebo response can be modified by color, size and preparation of pill. ${ }^{7}$ In this sense, the portion of response attributable to this inert (and thus non-specific) therapy can be called a "placebo effect." Nonetheless, delineating the boundaries of this definition remain difficult, as the scope of research expands beyond the inert pill (the comparator) toward an exploration into the meaning and effects of therapy in general. ${ }^{8}$

Regardless of its definition and scope, placebo effect does not influence the placebo response rate alone. The natural history of disease can influence placebo response separate from placebo effect, and irritable bowel syndrome is characterized by a fluctuating course. For example, $58 \%$ of patients with post-infectious IBS entered complete remission within six years of onset in one cohort study, and only $39 \%$ of patients met Rome III criteria 10 years after an initial diagnosis of IBS by Rome III criteria in a large ten-year longitudinal trial. ${ }^{10}$

Another effect known as the regression of symptoms toward mean severity is represented by an initial extreme measurement of IBS symptoms which corresponds with a second measurement of symptoms closer to the mean. Studies often wish to enroll the sicker patient to see a greater response. The problem in IBS is that some weeks are worse than others. If the study selects patients in the worst week of their month, they almost predestined to improve. This difference is reported in therapeutic response and unintentionally mistaken for efficacy. ${ }^{11}$ Adding a third no-treatment arm to clinical trials has been suggested to correct for these influences and elicit the true placebo effect from the overall placebo response rate. In cases where this would be unfeasible, conducting two baseline sequential evaluations of disease severity could track the magnitude of regression to the mean.

A multitude of unknown confounding factors in determining placebo response limit the generalizability of results. A recent meta-analysis of clinical trials including cross-over studies in IBS revealed that placebo response was higher in European trials than in US trials of pharmacologic therapies. ${ }^{3}$ Interestingly, there was no statistically significant difference when comparing trials conducted in secondary or tertiary care settings or with a single- or multi-center design. These data suggest the potential for a cultural influence on placebo effect, though what this is remains unknown and probably complex. For example, German studies of sham acupuncture revealed a correlation with expectation of benefit while a well-known US trial did not. ${ }^{12}$ Further efforts are needed to rectify conflicting results among the many individual trials.

\section{Current Methods of Reducing Placebo Response}

One strategy to reduce high placebo response is to predict and directly attempt to control the placebo response of patients entering clinical trials. A run-in phase is typically employed to weed out high-responders to placebo, with longer run-in phases associated with lower placebo response. ${ }^{13,14}$ However, the validity of employing run-in phases is not always clear as results may not provide meaningful real-world conclusions. ${ }^{15}$ Assessment of emotional state prior to study appears to be important in studies of IBS. This effect appears to be bidirectional, as higher anxiety states may contribute to reduced placebo response ${ }^{16}$ and lower anxiety states raise placebo response. ${ }^{17}$ Frequency of intervention correlates inversely with higher global improvement scores, thus therapies administered once daily rather than 2 or 3 times may reduce placebo response. ${ }^{14}$ Finally, clinicians who frequently assess patients and are more caring in their interactions may elicit a higher placebo response. ${ }^{18,19}$ While all of these strategies can reduce placebo response (Table), the concept of limiting physician-patient interaction and prescribing low frequency therapy which is suboptimal based on pharmacokinetics might not be appropriate in a real-world setting.

\section{Neurobiological Approaches to Managing Placebo Effect}

A number of neurobiologic theories of placebo effect have been explored. Support for an opioidergic pathway derives from a large body of literature reporting evidence of reversal of placebo analgesia with an opioid antagonist. ${ }^{20}$ This has been shown in a

Table. Traditional Methods of Reducing Placebo Effect in Clinical Trials

Run-in phase preceding randomization to identify high placebo response

Comparative assessment of baseline depression and anxiety with a validated scale

Lower frequency of intervention

Optimizing and standardizing patient-physician relationships 
recent operant rat model based on conditioning of a placebo response $^{21}$ and human studies. ${ }^{22}$ Based on functional MRI, areas of the central nervous system have been identified which appear to be involved in placebo analgesia in response to rectal distention. ${ }^{23}$

However, a separate study identified differences in regional brain activity based on functional imaging of patients receiving either saline placebo or opioid, suggesting the presence of a non-opioid pathway in placebo analgesia. ${ }^{24}$ While many studies have been carried out in an IBS population, evidence supporting this pathway is generally based on the study of placebo analgesia. Whether this pathway plays a significant role in the IBS placebo response was controversial in one small study in which naloxone did not blunt Likert-based ratings of placebo analgesia of an IBS cohort which underwent rectal distention. ${ }^{25}$

A second potential and possibly concurrent pathway is based on placebo-mediated endogenous dopamine release which is degraded by catechol-O-methyltransferase. ${ }^{26}$ Recent efforts have identified the catechol-O-methyltransferase Val158Met polymorphism as a predictor of placebo response based on increased degradation of the variant protein. ${ }^{27}$ This study found a statistically significant reduction in "active" placebo response which was not seen among no-treatment ("wait-list") controls. This demonstrated a placebo-specific response. A clear advantage of this strategy is that it does not require major changes in trial design and can therefore lead to near-term improvement in reducing placebo effect.

\section{Psychological Approaches to Managing Placebo Effect}

A number of psychological theories to explain placebo effect have been put forth, including expectation of improvement, conditioning of response based on prior consistent therapy with an active modality followed by replacement of the active therapy with placebo, ${ }^{28}$ and emotional state. Expectancy theory involves a sense of achieving forthcoming improvement which precedes the initiation of therapy in the clinical trial. Conditioning theory is based on the unconditioned sense of improvement becoming conditioned with the application of (conditioned) effective drug therapy which can then be replaced by placebo. The relative contribution of these theories remains somewhat controversial though not mutually exclusive. ${ }^{29}$ Similarity between successfully conditioned response and expectancy has been identified and validated using functional MRI which reveals similar brain activity. $^{30}$

\section{Expectancy and the Pre-cebo Effect}

The majority of studies have focused on expectation of improvement which can be affected by patient recruitment and communication prior to study initiation given that patients have generally never previously received the experimental therapy. ${ }^{31}$ The magnitude of the expectancy effect was apparent in a withdrawal study in which patients were injected with analgesia either in plain view or without the patient's knowledge in a cohort of healthy patients with experimentally induced pain and a second cohort of post-operative patients. In this evaluation, hidden injection resulted in significantly less pain relief in both experiments. ${ }^{32}$ This is also supported by meta-analyses revealing that placebo response rates are higher in trials of placebo analgesia (with placebo as an "active" therapy) compared to lower placebo response rates in placebo-controlled drug trials. ${ }^{33}$

Expectancy does not appear to be an "on-off” phenomenon. It may instead influence placebo response in a bidirectional manner and therefore contribute to nocebo response, or expected worsening leading to poor endpoint response. Based on an opioidergic mechanism, pain tolerance was blunted in one study when patients were deceptively informed that they were receiving placebo. ${ }^{34}$ The same authors conducted an evaluation of similar design to evaluate a dopaminergic mechanism in which Parkinson disease patients underwent a neurosurgical procedure to stimulate the subthalamic nuclei. Motor performance decreased in patients who were deceptively informed that this device had been "turned off." While the ethics of untrue statements to patients limits general application of these principles, expectancy may still develop among subjects without intervention. Kotsis et $\mathrm{al}^{35}$ conducted a study in which healthy patients were deceptively informed that they had a $50 \%$ chance of receiving analgesia when all patients actually received normal saline. Despite the application of the same therapy in all patients, those who believed they were receiving analgesia had significantly lower pain scores when induced by rectal balloon than subjects who believed they were receiving placebo. ${ }^{35}$

The results of these experimental models based on placebo analgesia have correlated with analogous experiments in an IBS population. In one study comparing IBS and healthy cohorts, the authors either informed patients that they would receive a novel analgesic therapy with rapid onset and short duration, or that they would receive saline, prior to rectal distention. Placebo analgesia was similar between placebo and control as well as IBS and healthy populations. ${ }^{16}$ These effects do not account for the entirety of placebo response. In a recent trial of open-label "inert" 
placebo against a no-treatment arm in IBS patients, patients obtained statistically significant benefit on placebo therapy. ${ }^{36}$ Despite these findings, reproducibility of placebo analgesia results in IBS appears limited by familiarity with study methodology based on decreasing subsequent unpleasant sensation of rectal distention in a trial of IBS and healthy controls over 12 months. $^{37}$

Our group recently published a study revealing a significant contribution of expectancy toward placebo effect and subsequent trial results in IBS. ${ }^{38}$ This finding was based on the influence of pre-study communication which can be defined as the pre-cebo effect. With the lack of a gold standard for the design of informed consent forms, specific wording can affect study results with a varying degree of bias due to pre-conceived notions of efficacy of the various drug classes. ${ }^{39}$ Patients were randomized to one of three questionnaires based on language from actual consent forms used in trials of desipramine, alosetron and rifaximin to explain the study protocol (including dosing frequency and treatment length), how the drug worked, and potential effect of the drug on IBS which justified the purpose of the trial. Patients were then asked the percentage of improvement they would have to attain to say that they achieved "adequate relief of symptoms." Patients reading the consent language for desipramine and alosetron reported that they would have to attain $73 \%(P<0.01$ against rifaximin results by one-way ANOVA) and 76\% ( $P=0.049$ against rifaximin results) improvement, respectively, compared to $87 \%$ improvement on rifaximin. In contrast to antidepressant therapy, we postulate that the idea of an antibiotic only somewhat relieving an illness does not correlate with the public's view of their curative nature. ${ }^{40}$ One weakness of this initial study was that patients were informed that this was a mock trial and there would be no therapy. A prospective trial evaluating various consent language followed by deceptive therapy with the same investigational agent could confirm these results.

\section{Conditioning and the Unblinding of Side Effects}

Traditional Pavlovian conditioning of a placebo response occurs after drug response is initially achieved and similar therapy - active or not - is continued. This was shown using a modified model of expectancy in which pain was induced using a tourniquet with subsequent administration of either opioid or saline. Study subjects were then conditioned with ketorolac until the final day, upon which all patients received saline instead of ketorolac to measure the conditioned response. Those conditioned with ketorolac only showed partial reversal with subsequent naloxone therapy, suggesting a conditioned response. ${ }^{41}$ Despite the seemingly subconscious nature of this theory, conditioning may not occur after replacing active therapy with placebo if baseline disease severity approximates conditioned disease severity. Therefore, conditioning may be a learned response requiring a consciously palpable subjective improvement in disease presentation. ${ }^{42}$ Given the need for initial improvement which can propagate a conditioned response, it is reasonable that a conditioned response could also endure with continued active drug therapy.

The extensive side effect profiles of some IBS treatment modalities may lead to spontaneous unblinding to active therapy in clinical trials, which may in turn impact trial outcome. We recently evaluated the known side effect profiles of therapies for IBS which received at least a Grade $1 \mathrm{~b}$ rating from the American College of Gastroenterology (ACG) Task Force using chi-square analysis to evaluate the correlation between incidence of adverse events (on active therapy relative to placebo) and efficacy based on traditional subjective endpoints. ${ }^{43}$ Interestingly, we found that higher adverse event incidence on active therapy was associated with more beneficial patient-reported outcome in IBS clinical trials. ${ }^{44}$ This suggests a positive conditioning effect which we attribute to a higher sense of "potency."

\section{The Elusive Endpoint}

The aforementioned strategies can reduce placebo response rates directly, but whether drug response rates are concurrently affected remains uncertain but probable. Rather than focusing on optimizing placebo, the majority of energy has been focused on improving outcome measures in IBS. For years, the traditional endpoint in IBS was adequate relief of abdominal pain and discomfort. $^{45}$ Slightly different endpoints of adequate relief of global symptoms or a Likert-scale measuring subjective global relief were subsequently developed and used in many initially successful IBS drug trials. ${ }^{46}$ These endpoints were in line with recommendations from the Rome III committee toward the use of binary primary endpoints based on patient-reported measures. ${ }^{47}$

\section{Patient-reported Outcomes}

Patient-reported outcomes have remained prevalent in IBS trials ${ }^{48}$ based on physician-based outcomes showing only weak to moderate (though statistically significant) correlation with patient-reported outcomes in functional gastrointestinal disease. ${ }^{49}$ One study highlighted the paradoxical inverse relationship be- 
tween binary satisfactory relief and baseline symptom severity. ${ }^{50}$ This later proved controversial, as exclusion of patients with baseline relief appeared to alleviate this concern. ${ }^{51}$ Regardless, the binary patient-reported assessments of adequate and subjective relief remain validated against psychometric properties. ${ }^{52}$

Given the breadth of symptoms listed in consensus-based clinical criteria for IBS, ${ }^{47}$ a major concern with patient-reported outcomes is the high placebo response rate which may be correlated with placebo analgesic effects. A recent evaluation of patient-reported outcomes in asthma revealed similar findings which were compared against objective and well-validated measurements of forced expiratory volume in 1 second (FEV1). ${ }^{53}$ Patients were administered albuterol, sham acupuncture, or no intervention and asked to rate their improvement as well as undergo measurement of FEV1. While there was significant improvement on albuterol based on FEV1 measurement, there was no benefit to albuterol with a self-reported endpoint which highlights the ongoing need for objective data to conduct similar to IBS validation trials.

\section{Concerns With Existing Binary Patient-based Endpoints}

Two of the most positive characteristics of binary subjective patient endpoints are that these endpoints strictly evaluate whether a therapy is "working" and also account for differences in culture and values. Within this construct, there is unfortunately no built-in method with which to weigh a positive outcome with an undesirable one. Therefore this assessment of a complex mix of complaints and sensations summed into a single response may be inadequate. Therapies which are poorly tolerated or which cause relatively frequent significant adverse events may still lead to significant adequate relief of IBS relative to placebo. Famously, adequate relief of global IBS symptoms was used to evaluate alosetron which later was withdrawn due to constipation as an adverse event presumably leading to ischemic colitis. ${ }^{54,55}$

One method to account for the overall harm of therapy is "dropouts due to adverse events," implying that a patient (or physician as the patient's agent) sees greater definite harm with continuing therapy than returning to baseline IBS severity even in light of potential drug benefits. This measure also accounts for differing culture and values in that the patient must weight benefit and risk. We conducted a meta-analysis ${ }^{56}$ evaluating IBS therapies which received at least a Grade $1 \mathrm{~b}$ from the ACG Task Force for IBS primary endpoints ${ }^{43}$ for dropouts due to adverse events. For diarrhea-predominant IBS (IBS-D), antidepressant and alosetron therapy led to one dropout due to adverse events for only every 2.3 and 2.6 patients benefiting from therapy, respectively. In contrast, for every 846 patients benefiting from rifaximin therapy, only one patient dropped out due to adverse events and this was not statistically significant. This was in contrast to rifaximin having the lowest reported number needed to treat in the literature based on patient-reported binary endpoints. For constipation-predominant IBS (IBS-C), lubiprostone appeared safe and there were not enough data to evaluate selective serotonin reuptake inhibitors. These data suggest the importance of a harm evaluation concurrently with an efficacy evaluation in IBS and point to the continued inadequacies of existing outcome measures.

\section{Recent Updates in Outcome Design}

In response to concerns with binary patient-based outcomes, the Food and Drug Administration (FDA) released 2 endpoints for IBS based on the underlying motility complaint rather than a single endpoint encompassing IBS in general. These most recent endpoints incorporated an objective measure of stool frequency into the binary outcome of abdominal pain and discomfort. The European Medicines Agency (EMA) recommended co-endpoints of global improvement and specific improvement in abdominal discomfort or pain which can still identify similar percentages of responders compared to subtype-based FDA endpoints. ${ }^{48}$ Despite controversy surrounding therapies which are aimed at IBS in general regardless of subtype, the EMA is considering development of separate endpoints based on the underlying motility complaint. ${ }^{57}$

The modified FDA endpoint for IBS-C was implemented in two phase III trials published last year which supported the use of linaclotide in treating IBS, a secretagogue therapy which accelerates gut motility. ${ }^{58-60}$ This new endpoint incorporates a binary measure of complete spontaneous bowel movements which still does not evaluate whether a patient decides that this increase in bowel movements is an adverse event. Based on data from the linaclotide trials, this IBS-C endpoint was validated against patient rating-of-change questions asked concurrently with the drug trials. $^{61}$ Despite this, more patients on therapy suffered diarrhea as an adverse event than they did improve based on the new endpoint. In an update to our previously described meta-analysis of harm, linaclotide only benefited 2.6 patients for every patient dropping out due to adverse events based on recently modified endpoints and mostly due to diarrhea. Current IBS endpoints appear to insufficiently evaluate overall change in motility.

Our group recently conducted an analysis of intervention 
group relative to placebo in which we compared the proportion of patients meeting endpoint to the proportion suffering the motility side effect opposite their underlying complaint. ${ }^{62}$ For example, is it desirable to take a patient with IBS-D and make them constipated? By incorporating this measure of motility into the standard subjective endpoint analysis of efficacy, "functional net value" was determined to represent the net percentage of patients achieving remission of disease rather than a swing of the pendulum to the opposite motility event. Rifaximin, a non-absorbable antibiotic still under evaluation for treating IBS-D, did not cause constipation in eligible clinical trials. Alosetron, now restricted to women with severe refractory IBS-D, ${ }^{63}$ was found to have a large negative functional net value despite its meeting subjective primary endpoints in clinical trials. However, linaclotide led to the opposite motility complaint more frequently than did alosetron. A measure such as functional net value suggests that a motility-based endpoint may be better suited for a disease characterized by motility complaints.

\section{Conclusions}

In sum, IBS is characterized by abdominal pain and discomfort but also by deranged motility which is not well measured in clinical trials. Placebo therapy can alter subjective outcome measures and confound results, but novel methods have been described which could help minimize the placebo effect in clinical trials. Outcomes of trials in the past decade did not predict the fate of some therapies which reached market. However, strong efforts are being made to improve clinical trial design in IBS that should lead to improved and more accurate evaluations of therapy.

\section{References}

1. Bernstein $\mathrm{CN}$. The placebo effect for gastroenterology: tool or torment. Clin Gastroenterol Hepatol 2006;4:1302-1308.

2. Enck P, Bingel U, Schedlowski M, Rief W. The placebo response in medicine: minimize, maximize or personalize? Nat Rev Drug Discov 2013;12:191-204.

3. Ford AC, Moayyedi P. Meta-analysis: factors affecting placebo response rate in the irritable bowel syndrome. Aliment Pharmacol Ther 2010;32:144-158.

4. Beecher HK. The powerful placebo. J Am Med Assoc 1955;159: 1602-1606.

5. Hróbjartsson A, Gøtzsche PC. Placebo interventions for all clinical conditions. Cochrane Database Syst Rev 2010;(1):CD003974.

6. Duracinsky M, Chassany O. [How can an effective drug to treat irritable bowel syndrome be successfully developed?]. Gastroenterol
Clin Biol 2009;33(suppl 1):S26-S34. [French]

7. Kienle GS, Kiene H. Placebo effect and placebo concept: a critical methodological and conceptual analysis of reports on the magnitude of the placebo effect. Altern Ther Health Med 1996;2:39-54.

8. Moerman DE, Jonas WB. Deconstructing the placebo effect and finding the meaning response. Ann Intern Med 2002;136:471-476.

9. Marshall JK, Thabane M, Garg AX, et al. Eight year prognosis of postinfectious irritable bowel syndrome following waterborne bacterial dysentery. Gut 2010;59:605-611.

10. Olafsdottir LB, Gudjonsson H, Jonsdottir HH, Thjodleifsson B. Stability of the irritable bowel syndrome and subgroups as measured by three diagnostic criteria - a 10-year follow-up study. Aliment Pharmacol Ther 2010;32:670-680.

11. Morton V, Torgerson DJ. Regression to the mean: treatment effect without the intervention. J Eval Clin Pract 2005;11:59-65.

12. Witt CM, Schützler L. The gap between results from sham-controlled trials and trials using other controls in acupuncture research - the influence of context. Complement Ther Med 2013;21:112-114.

13. Patel SM, Stason WB, Legedza A, et al. The placebo effect in irritable bowel syndrome trials: a meta-analysis. Neurogastroenterol Motil 2005;17:332-340.

14. Pitz M, Cheang M, Bernstein CN. Defining the predictors of the placebo response in irritable bowel syndrome. Clin Gastroenterol Hepatol 2005;3:237-247.

15. Berger VW, Rezvani A, Makarewicz VA. Direct effect on validity of response run-in selection in clinical trials. Control Clin Trials 2003; 24:156-166

16. Lee HF, Hsieh JC, Lu CL, et al. Enhanced affect/cognition-related brain responses during visceral placebo analgesia in irritable bowel syndrome patients. Pain 2012;153:1301-1310.

17. Morton DL, Watson A, El-Deredy W, Jones AK. Reproducibility of placebo analgesia: Effect of dispositional optimism. Pain 2009;146: 194-198.

18. Kelley JM, Lembo AJ, Ablon JS, et al. Patient and practitioner influences on the placebo effect in irritable bowel syndrome. Psychosom Med 2009;71:789-797.

19. Dorn SD, Kaptchuk TJ, Park JB, et al. A meta-analysis of the placebo response in complementary and alternative medicine trials of irritable bowel syndrome. Neurogastroenterol Motil 2007;19:630-637.

20. Levine JD, Gordon NC, Fields HL. The mechanism of placebo analgesia. Lancet 1978;2:654-657.

21. Nolan TA, Price DD, Caudle RM, Murphy NP, Neubert JK. Placebo-induced analgesia in an operant pain model in rats. Pain 2012;153:2009-2016.

22. Benedetti F. The opposite effects of the opiate antagonist naloxone and the cholecystokinin antagonist proglumide on placebo analgesia. Pain 1996;64:535-543.

23. Eippert F, Bingel U, Schoell ED, et al. Activation of the opioidergic descending pain control system underlies placebo analgesia. Neuron 2009;63:533-543.

24. Petrovic P, Kalso E, Petersson KM, Andersson J, Fransson P, Ingvar M. A prefrontal non-opioid mechanism in placebo analgesia. Pain 2010;150:59-65.

25. Vase L, Robinson ME, Verne GN, Price DD. Increased placebo analgesia over time in irritable bowel syndrome (IBS) patients is associated with desire and expectation but not endogenous opioid 
mechanisms. Pain 2005;115:338-347.

26. de la Fuente-Fernández R. The placebo-reward hypothesis: dopamine and the placebo effect. Parkinsonism Relat Disord 2009;15 (suppl 3):S72-S74.

27. Hall KT, Lembo AJ, Kirsch I, et al. Catechol-O-methyltransferase val158met polymorphism predicts placebo effect in irritable bowel syndrome. PLoS One 2012;7:e48135.

28. Bryant CD, Roberts KW, Culbertson CS, Le A, Evans CJ, Fanselow MS. Pavlovian conditioning of multiple opioid-like responses in mice. Drug Alcohol Depend 2009;103:74-83.

29. Stewart-Williams S, Podd J. The placebo effect: dissolving the expectancy versus conditioning debate. Psychol Bull 2004;130:324-340.

30. Watson A, El-Deredy W, Iannetti GD, et al. Placebo conditioning and placebo analgesia modulate a common brain network during pain anticipation and perception. Pain 2009;145:24-30.

31. Rutherford BR, Roose SP. A model of placebo response in antidepressant clinical trials. Am J Psychiatry 2013;170:723-733.

32. Amanzio M, Pollo A, Maggi G, Benedetti F. Response variability to analgesics: a role for non-specific activation of endogenous opioids. Pain 2001;90:205-215.

33. Vase L, Riley JL 3rd, Price DD. A comparison of placebo effects in clinical analgesic trials versus studies of placebo analgesia. Pain 2002; 99:443-452.

34. Benedetti F, Pollo A, Lopiano L, Lanotte M, Vighetti S, Rainero I. Conscious expectation and unconscious conditioning in analgesic, motor, and hormonal placebo/nocebo responses. J Neurosci 2003;23: 4315-4323.

35. Kotsis V, Benson S, Bingel U, et al. Perceived treatment group affects behavioral and neural responses to visceral pain in a deceptive placebo study. Neurogastroenterol Motil 2012;24:935, e462.

36. Kaptchuk TJ, Friedlander E, Kelley JM, et al. Placebos without deception: a randomized controlled trial in irritable bowel syndrome. PLoS One 2010;5:e15591.

37. Naliboff BD, Berman S, Suyenobu B, et al. Longitudinal change in perceptual and brain activation response to visceral stimuli in irritable bowel syndrome patients. Gastroenterology 2006;131:352-365.

38. Kim SE, Kubomoto S, Chua K, Amichai MM, Pimentel M. "Pre-cebo": an unrecognized issue in the interpretation of adequate relief during irritable bowel syndrome drug trials. J Clin Gastroenterol 2012;46:686-690.

39. Britten N. Patients' ideas about medicines: a qualitative study in a general practice population. Br J Gen Pract 1994;44:465-468.

40. Macfarlane J, Holmes W, Macfarlane R, Britten N. Influence of patients' expectations on antibiotic management of acute lower respiratory tract illness in general practice: questionnaire study. BMJ 1997;315: 1211-1214.

41. Amanzio M, Benedetti F. Neuropharmacological dissection of placebo analgesia: expectation-activated opioid systems versus conditioning-activated specific subsystems. J Neurosci 1999;19:484-494.

42. Montgomery GH, Kirsch I. Classical conditioning and the placebo effect. Pain 1997;72:107-113.

43. American College of Gastroenterology Task Force on Irritable Bowel Syndrome, Brandt LJ, Chey WD, et al. An evidence-based position statement on the management of irritable bowel syndrome. Am J Gastroenterol 2009;104(suppl 1):S1-S35.

44. Hani AA, Shah ED, Pimentel M. Sa1158 The number of drug side effects is associated with greater efficacy in IBS clinical trials: the potential influence of unblinding. Gastroenterology 2013;144(supple 1): S-217.

45. Mangel AW, Hahn BA, Heath AT, et al. Adequate relief as an endpoint in clinical trials in irritable bowel syndrome. J Int Med Res 1998;26:76-81.

46. Müller-Lissner S, Koch G, Talley NJ, et al. Subject's Global Assessment of Relief: an appropriate method to assess the impact of treatment on irritable bowel syndrome-related symptoms in clinical trials. J Clin Epidemiol 2003;56:310-316.

47. Drossman DA. The functional gastrointestinal disorders and the Rome III process. Gastroenterology 2006;130:1377-1390.

48. Corsetti M, Tack J. FDA and EMA end points: which outcome end points should we use in clinical trials in patients with irritable bowel syndrome? Neurogastroenterol Motil 2013;25:453-457.

49. Fallone CA, Guyatt GH, Armstrong D, et al. Do physicians correctly assess patient symptom severity in gastro-oesophageal reflux disease? Aliment Pharmacol Ther 2004;20:1161-1169.

50. Whitehead WE, Palsson OS, Levy RL, Feld AD, VonKorff M, Turner M. Reports of "satisfactory relief" by IBS patients receiving usual medical care are confounded by baseline symptom severity and do not accurately reflect symptom improvement. Am J Gastroenterol 2006;101:1057-1065.

51. Passos MC, Lembo AJ, Conboy LA, et al. Adequate relief in a treatment trial with IBS patients: a prospective assessment. Am J Gastroenterol 2009;104:912-919.

52. Spiegel B, Camilleri M, Bolus R, et al. Psychometric evaluation of patient-reported outcomes in irritable bowel syndrome randomized controlled trials: a Rome Foundation report. Gastroenterology 2009; 137:1944-1953, e1-e3.

53. Wechsler ME, Kelley JM, Boyd IO, et al. Active albuterol or placebo, sham acupuncture, or no intervention in asthma. N Engl J Med 2011;365:119-126.

54. Camilleri M, Mayer EA, Drossman DA, et al. Improvement in pain and bowel function in female irritable bowel patients with alosetron, a 5- $\mathrm{HT}_{3}$ receptor antagonist. Aliment Pharmacol Ther 1999;13:11491159.

55. Camilleri M, Northcutt AR, Kong S, Dukes GE, McSorley D, Mangel AW. Efficacy and safety of alosetron in women with irritable bowel syndrome: a randomised, placebo-controlled trial. Lancet 2000; 355:1035-1040.

56. Shah E, Kim S, Chong K, Lembo A, Pimentel M. Evaluation of harm in the pharmacotherapy of irritable bowel syndrome. Am J Med 2012;125:381-393.

57. Committee for Medicinal Products for Human Use (CHMP). Concept paper on the revision of the CHMP points to consider on the evaluation of medicinal products for the treatment of irritable bowel syndrome (CPMP/EWP/785/97). European Medicines Agency, May 2012. Available from URL: http://www.ema.europa. eu/docs/en_GB/document_library/Scientific_guideline/2012/06/ WC 500128217.pdf (accessed 23 January, 2014).

58. Center for Drug Evaluation and Research (CDER). Guidance for industry: irritable bowel syndrome - clinical evaluation of drugs for treatment. Food and Drug Administration, May 2012. Available from URL: http://www.fda.gov/downloads/Drugs/Guidances/UCM205269. pdf (accessed 23 January, 2014). 
59. Rao S, Lembo AJ, Shiff SJ, et al. A 12-week, randomized, controlled trial with a 4-week randomized withdrawal period to evaluate the efficacy and safety of linaclotide in irritable bowel syndrome with constipation. Am J Gastroenterol 2012;107:1714-1724.

60. Chey WD, Lembo AJ, Lavins BJ, et al. Linaclotide for irritable bowel syndrome with constipation: a 26-week, randomized, double-blind, placebo-controlled trial to evaluate efficacy and safety. Am J Gastroenterol 2012;107:1702-1712.

61. Macdougall JE, Johnston JM, Lavins BJ, et al. An evaluation of the
FDA responder endpoint for IBS-C clinical trials: analysis of data from linaclotide Phase 3 clinical trials. Neurogastroenterol Motil 2013; 25:481-486.

62. Shah ED, Pimentel M. 1039 Functional Net Value (FNV): an Important Consideration in Clinical Evaluation of IBS Pharmacotherapy. Gastroenterology 2012;142(suppl 1):S-182.

63. Lucak SL. Optimizing outcomes with alosetron hydrochloride in severe diarrhea-predominant irritable bowel syndrome. Therap Adv Gastroenterol 2010;3:165-172. 